effects follow and the tumour is reduced, then the inference is that the disorder was chronic inflammation or enlargement of the ovary, without serious change in structure.

Among mesenteric enlargements some yield readily to the use of the waters. Others are stubborn and resist these solvent agents as they resist all other measures. M. Petit states that he has seen various instances of the prompt resolution of mesenteric tumours under the use of these waters; and these tamours he had reason to think were epiploic or omental. If so we doubi whether these ought to be referred to the head of mesen. teric enlargements. Probably they ought to be denominated merely abdominal.

Vesical catarrh or catarrh of the bladder is another malady in which these waters are represented to be beneficial and therapeutic. The efficacy of the remedy he allows to depend upon the duration of the disorder and its severity; recent cases being always more easily removed than those of long standing, and mild cases than those of greater severity. In other words, cases, which are curable, are mild; cases, which are not curable, are severe and inveterate. Under all circumstances it appears that the remedy affords relief. The mere warm bath, indeed, is beneficial in this form of vesical disease; and the alkaline warm bath is likely to be still more decidedly so. One season at Vichy is rarely sufficient for cure. Several seasons are usually required.

Here, however, for the present it is necessary to pause. The author is certainly a strenuous advocate for the employment of the waters of Vichy; and we do not say that he is an indiscriminate one. Taken altogether, and attentively studied, his directions are probably as specific and as pointed as the subject admits. At the same time it is not difficult to perceive, that much of the knowledge hitherto acquired on the use of these waters in the treatment of disease is empirical; and that in a large proportion of cases the advice must be tentative.

ART. II,-1. Observazions on Aueurism and its Treatment by Compression. By O'Bryen Bellingham, M.D., Edinburgh, Fellow of, and Professor in, the School of the Royal College of Surgeons in Ireland, \&c. London, 1847, 12mo, pp. 181.

ART. HI. 2. Practical Remarks on the Treatment of Aneurism by Compression, with Plates of the Instruments hitherto employed in Dublin, and the recent Improvements by Elastic 
Pressure. By Jolliffe Tufnell, M. R. I. A., Fellow of the Royal College of Surgeons in Ireland; Surgeon to the City of Dublin Hospital; Surgeon to the Dublin District Military Prison; and Lecturer on Military Surgery in Dublin. Dublin, 1851, 8vo. pp. 154.

ALL the great operations of surgery have passed through various stages of rudeness, complexity, and inefficiency, and not unfrequently barbarism and cruelty, until they have attained what the surgeons of the day term elegance, simplicity, comparative efficiency, and some degree of mildness and humanity. Nor is the operation for aneurism of the large arteries by any means an exception. Whoever reads the histories recorded by some of our older surgeons of the mode, in which they cut open aneurismal tumours, the violent measures which they employed to arrest the gush of blood from the interior of the sac, the scooping out of clots, and the cramming the interior of it with charpee, sponges, and compresses, the long and tedious suppurations which thence resulted, the sloughing of soft parts, and the exfoliation of bones, the relentless use of hot irons and hot liquids, and the small number of instances in which patients escaped with life, and the not less small number, in which they escaped with limb, will not fail to admit, that, while surgery then required operators strong to inflict and patients stubborn to endure, the operation for aneurism in these times was neither bloodless, nor painless, nor void of danger, and offered at least only an alternative of the mode of extinction. It seemed, indeed, of little moment whether the patient died of the slow but sure approaches of the disease, or the more prompt measures of the surgeon. Such was the practice of Philagrius; and at a period long subsequent, though with certain modifications, of DE LA VAUGYON, LEGER DE GouEY, Ambrose Pare, ${ }^{*}$ Wiseman, $\uparrow$ Dionis, $\ddagger$ and it may be added, Daniel Turner. $\$$

Contemporary with Philagrius was Antyllus, who is represented to have introduced the use of the ligature, or the needle and ligature; but the employment of this measure was attended with neither benefit to the patient nor substantial improvement of the operation. ANTYLlus did not, like modern surgeons, dis-

- The Works of that Famous Chirurgeon, A mbrose Parey, translated out of l.atin and compared with the French. By Th. Johnson. London, 1678. Polio, Book VII., chapter xxxii, p. 194.

+ Several Surgical Treatises. By Kichard Wiseman, Sergeant-Surgeon. London, 1676. Small folio, Book I., chapter xvi, pp. 72, 73, 76, \&c.; and Book chapter ii., p. 355.

\$ Cours d'Operations de Chirurgie de montrées au Jardin Royal. Par M. Dionis. 4ieme edition. Par G. De la Faye A Paris, 1740. 8vo Huitieme Demonstration, p. 688, \&c.

$\$$ The Art of Surgery. In two volumes. By Daniel Turner, M D., of the College of Physicians in London. The sixth edition correeted. London, 1741. Tol. 1., section v., p. y47. Treatment by compression, by operation, 548 , \&ce. $V$ dau 8 
sect, expose, and isolate the artery, which was to be enclosed and commanded by the ligature. He thrust the needle armed with two ligatures through the skin, deep into the muscles and soft parts of the limb, usually the arm, and, bringing out the needle with the ligature, he tied the whole parts, including the vessel, above and below the tumour, opened the aneurismal sac, cleared out the clots of blood, and filled it with digestive and suppurative applications. These were the days of what is called heroic Surgery.

Little improvement upon this rude method of procedure was the use of the actual cautery, as recommended and practised by Lanfranc; and after him by Mark Gatinara, Durando Scacchi, and Gabriel Failopius, the last of whom further states that he successfully employed caustics, we suppose the potential or chemical, in order to promote suppuration in the sac. Marcus Aurelius Severinus is said to have been the first surgeon, who applied a ligature to the crural artery in an aneurisinal condition, very near the ligament of Poupart.

Till the sixteenth century, therefore, the treatment of aneurism consisted in inclosing rudely in ligature the ingredient and sometimes the egredient arteries, clearing out the sac, and effecting suppuration and granulation; and in some rare cases in the entire removal of the tumour by incision. Not the smallest of the evils of this mode of operation was, that often it left the patient and his disease in a worse condition than that in which it found both.

Even when the aneurismal tumour was laid open and duly crammed with compresses according to the rules of art, removal of the limb by amputation was a second resource not unfrequent.

Under these circumstances, it is not wonderful that any practicable or probable method of curing the disease in a manner less bloody, less painful, and less tedious, should be received with some favour. JoHN DE VIGo is generally understood to have proposed, about the beginning of the sixteenth century, the use of graluated compression and styptic or astringent applications.* The same method Dura ndo Scacchi wished to employ, at least in the first instance; and both FAlLopius and PARE appear to have employed pressure by compress and bandage in various instances of aneurism, but especially in that of the brachial artery when wounded instead of the vein. +

It must not be forgotten, also, that some surgeons at this period laid it down as a rule, ihat aneurisms of the extremities ought to be treated only by compression, and that no cutting instrument should be employed.

It is probable that this plan was followed by occasional success; for

* Practica Copiosa in Arte Chirurgia Libros Novem Complectens. Edita a Johnne Vigo. Julii 2di Pontificis Maximi Olim Chirurgico. Lugduni Impressa, 1518. Liber IV.. Part V., Folin exxiii.

to The Works of that Famous Chirurgeon, Ambrose Parey, translated out of Letin and compared with the French. By Th. Johnson. London. Folio, 1, 78. Book V II, chapter xxxii., p. 194. 
the Abbe Bourdelot, first physician to the Prince, is represented to have employed on his own arm, towards the middle of the seventeenth century, a sort of tourniquet in the cure of a false aneurism produced in undergoing venesection. This person applied over the tumour a small steel plate covered with cotton and leather, leaving only a sort of groove where the artery was expected to lie, and secured it firmly to the arm. This steel plate he wore for twelve months, and at the end of that time the cure was completed.*

Similar means of compression were employed by various surgeons in the course of this century $y_{5}$-by Scultetus, Tulpius, Petit, and others; and those most addicted to mechanical contrivances were sufficiently fertile in proposing various pieces of mechanism more or less ingenious, and more or less powerful, which should have the effect of compressing the tumour and the artery, obliterating the 1 atter, and causing the former to disappear.

The practice, nevertheless, was not uniform. LA Morte, who practised during the latter years of the seventeenth century and the beginning of the eighteenth, appears to have been partial to the use of the button of blue vitriol placed in the artery, and covered by dressings and compresses; and though it is clear, from his own account, that this practice was not always attended with good results, he evidently reposed in it considerable confidence.t It is singular that some surgeons appear to have assigned as a reason against the use of compression the fact, that it obliterates the artery. They appear to have been unaware, that scarcely can any aneurism be cured without obliteration of the artery, and that obliteration is the most common consequence of all successful methods of treating aneurism.

From the end of the seventeenth to the middle of the eighteenth century, the practice among surgeons may be regarded as represented by PETER DIONIs and his commentator DE LA Faye in France, and Laurence Heister and John $Z_{\text {acha- }}$ rias Plat wer in Germany. These three authorities agree in recommending, in the cure of aneurism of the extremities, compression in the first instance, and if this be not successful, the operation.

Particularly pertinent is the observation of M. De La Faye. When we cannot by compression, he says, cure an aneurism or prevent its progress, we, nevertheless, derive from this method great advantages. By compressing the vessel we prevent the blood from flowing into it in so great amount as ordinary. This obliges part of the blood to dilate gradually the collateral vessels,

- Dionis Cours D'Operations de Chirurgie, 4ieme edition. Par G. De La Faye. Paris, 1740. Huitieme Demonstration, p. 697.

+ La MotTe Traité Complet de Chirurgie. Par M. Gillaume Manquest de la Motte Chir. Juré. Troisiene Editions Revue, corrigée, et Augmentée de Notes Critiques, pars M. Sábatier. Prof. Roy. A Paris, 1771.8vo. Deux tornes. Tome Se cunde. Plaius des Extremités. Observ. 270 and 271, p. 182-188. 
and disposes them to supply the function of the principal artery, which it it proposed to enclose in a ligature. What is here advanced experience confirms. M. Petit has made to me the remark, that the operation for aneurism almost al ways succeeds, when it is performed after having long subjected the artery to compression.*

Heister mentions four different methods of treating aneurismal tumours ; first, by compression and bandaging, if small, as in the arm; secondly, in the instance of large aneurisms, by the operation of cutting into the sac, enclosing the artery in a ligature, and employing afterwards compression within the cyst until it was cicatrized; thirdly, the method of Purmann, in placing a ligature upon the artery above and below the tumour without touching the latter; and, fourthly, the method of Anel. $\dagger$

John Zaccharias Piattner, $\nmid$ a surgeon who flourished in the beginning of the eighteenth century, devoted great attention to the determination of the order of cases, in which the treatment of aneurism by compression is admissible, and to the improvement of the method of treatment. When the tumour is not of long duration and not very large, when it is situate in the arm or over the course of a bone, and is something circumscribed, he recommends that friction should be employed gently, in order to resolve the coagulated blood. He then applied over the tumour several folds of paper moistened in alcohol, and a graduated series of compresses with a piece of money, metal, or any other hard body between them, and applied over the whole a firm, retentive roller. He further recommends as serviceable, a compressing instrument invented by SENFF, a surgeon at Berlin, and which consisted of a pad worked by a screw, so as to direct its force upon the tumour. In traumatic aneurism, or that produced by blood-letting or other wound, he recommends the use of a leaden plate, covered by various compresses, and secured by a bandage, the apparatus being moistened with camphorated spirit. Blood-letting he regarded as serviceable in diminishing the afflux of blood to the tumour.

Notwithstanding this preference for the method by compression in certain circumstances, Plattner did not overlook the advantages of the ligature. He was in the habit of applying three ligatures round the aneurismal vessel; two above and one below the tumour, the last of which he believed to be necessary to prevent the influx of blood into the tumour by the collateral arteries. Between these ligatures and the artery he recommen-

- Dionis Cours d'Operations de Chirurgie, p. 695-note.

$\uparrow$ D. Laurentii Heisteri Institutiones Chirurgicae. Oyus Triginta Annorum. Pars. Prima et Secunda Amstelaedami 1739. Pars Altera, \&c. Sectio ii., cap. xiii., p. 440, \&c.

† Jo. Zachariae Plattneri, D. et Frof. Med. Lips. Institutiones Chirurgiae Rationalis Tum Medicae Tum Manualis in usus Discentium. Editio Altera Lirsiae, 1758, 12mo, pp. 944. Date of preface, Leipsic, $23 \mathrm{~d}$ May $1745, \S 431 .-\$ 443$. 
ded the interposition of a piece of linen, in nrder to avoid cutting the artery by the threads.

In these days the exact operation of the ligature was not understood.

Compression, nevertheless, had its advocates. Genga, a Roman surgeon, in the beginning of the eighteenth century, had been led to use a similar plan in treating both wounds and aneurisms of the brachial artery, which this surgeon was occasionally in the habit of opening, for the cure of certain severe attacks of headach. The method of GENGA was to apply a firm compress over the tumour or wound, and secure it by means of a firm uniform bandage, which was continued from the fingers the whole way up the arm. Carlo Guattani, the celebrated surgeon of Genoa, recommended the method strongly, and practised it with great success in the treatment of false aneurisms; applying over the tumour thick compresses, and retaining them by firm bandages. The true aneurism he continued to treat by ligature, especially when of long standing and considerable size.

Theden, a military surgeon of Prussia, of great celebrity in his day, contributed to render the practice of treatment by compression, serviceable if not popular. He was in the habit of applying over the tumour a plate of lead, and maintaining it in its position by a circular bandage or strap, while in order to prevent oedema and the irksomeness of partial local pressure, he enveloped the whole limb from its extremities upwards in broad long bandages carefully applied. This method by general compression, however, was still found occasionally inadequate to effect a cure, it was said, chiefly in aneurisms of long standing; and hence the operation in one shape or other was still frequently employed by surgeons.

One great objection to the operation appears, from the testimony of various surgeons at that time, to have been the circum. stance, that either by inattention the nerve was included in the ligature of the artery, or it was accidentally inclosed in the ligature; and to this ligature of the nerve various evils, and especially the final failure of the operation were ascribed. On this account, while John Macgill recommended the ligature as the most certain and efficacious method of curing aneurismal tumours, he particularly impressed the necessity of dissecting the artery and avoiding the ligature of the nerve; while Donald Monro, in order to insure the isolation of the artery, proposed to carry a probe into its interior, and thus raise it before tightening the ligature. Sharp, on the other hand, appears to have entertained less apprehension of enclosing the nerve in the ligature, than of lacerating or cutting the artery by its means.*

* A Treatise on the Operations of Surgery, \&c. By Samuel Sharp, Surgeon 
It is remarkable, that in these days, no dread appears to have been entertained of the effects of enclosing in the ligature the vein or any of its branches. It is next to certain, nevertheless, from the facts which modern experience, aided by dissection, have brought to light, that great mischief often resulted from ligature or cther injuries of veins, that these vessels were often attacked by inflammation after the operation, and that this venous inflammation must in many instances have proved one cause quite sufficient, without others, to bring about the fatal termination of the case. Tying both nerve and vein, and at least injuring much the latter, were almost unavoidable in any form of the old operation for aneurism; and when we consider the uncertain ideas that prevailed on the action of the ligature and the mode of its application, as well as the other modes of treating the sac, that failures were numerous and mortality considerable, are circumstances not in any way extraordinary.

At length John Hunter, by that superior power which distinguishes genius, devised a method of treatment which possessed the meritcertainly of being greatly more simple, infinitely less painful, scarcely bloody, in no comparison so tedious as that formerly in use, and which, by avoiding many avoidable causes of mischief, not only gave patients a much better chance of recovery, but did not so completely deprive them of those, which the presence of the aneurismal tumour had left. It was one great advantage of the method introduced by John Hunter, that, by placing the ligature upon the artery above the aneurismal tumour, and as remote from that tumour as the case allowed, he not only avoided touching parts greatly diseased in structure and deranged in relative position, but, by taking advantage of the restorative powers of the organic textures and the system at large, it gave an opportunity for the tumour being cured by the ordinary operation of those powers which are at all times at work in the living body.

The method proposed by John Hunter in 1785 was in general rather successful in this country in the hands of Sir Everard Home, Mr Abernethy, Sir Astley Cooper, Mr Lawrence, and Sir Benjamin Brodie. Abroad it seems to have failed in the bands of such eminent surgeons as Vacca, Fflajani, and others ; a eircumstance which probably is to be ascribed, in some degree, to the use of pieces of linen, tape, and broad ligatures, under the idea that the narrow thread cuts the artery, and thereby causes hemorrhage. Inflammation and sloughing of the arterial coats and consequent hemorrhage were the frequent and almost certain consequences of the application of the slips of linen, the tapes and similar

to Guy's Hospital. The Tenth Edition, with several alterations, London, 1751. 8vo. Chapter xxxvi. and xvxvii., p. 205.

A Critical Enquiry into the Present State of Surgery. By Samuel Starp, F.R.I., and Surgeon to Guy's Hospital, the Third Edition. London, 1754, 8vo, Chapter Eighth, p. $2980-313,311-313$, for his facts and arguments as to harmlessness of tying the nerve.

VOL. LXXVI. No. 188. 


\section{$218 \mathrm{Dr}$ Bellingham and $\mathrm{Mr}$ Tufnell on the Treatment of}

appliances, whether as safety ligatures (ligatures d'attente) or not.

It must not be denied, nevertheless, that in many instances of aneurism of different arteries of the extremities, though the operation was undertaken under circumstances considered favourable, and was accomplished with ability and dexterity, and with every precaution to insure a favourable issue, the result has been otherwise. The only mode in which the merits of any given operation can be determined has been adopted; namely, a careful statistical survey of all the recorded instances of operation and their results; and the result of this numerical examination has been to show, that the operation performed under all the most favourable conditions, and with due attention to all rules is, nevertheless, attended with an amount of mortality, which is considered as large.

The sources of information on this topic are three.-One is the work of Dr Crisp on diseases of the blood-vessels. In this treatise are given the particulars of one hundred and eighty-eight cases in which the artery was secured for popliteal or femoral aneurisin. Of these

Died from the effects of the operation,

Recovered after undergoing subsequent amputation,

Recovered after sloughing of the sac,

Recovered after mortification of the toes,

Recovered after sloughing of the integuments,

\section{5}

188

11

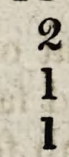

So that more than one-fourth of these 188 cases either terminated fatally or were maimed for the rest of life ; while one hundred and thirty-eight cases recovered.

Secondly, Mr Phillips collected one hundred and seventy-one cases of aneurism affecting the arteries of the lower extremities in which the Hunterian operation was performed. Of these cases fifty-seven, or, exactly, one in three, were unsuccessful, in which all the patients, except two, died, not of the disease but of the operation. Among the successful cases (114) secondary hemorrhage took place fifteen times. In fifty-nine of these cases the femoral artery was enclosed in a ligature; and in thirty-nine of these the results were unfavourable; thus giving a mortality of two in three in the artery most frequently subjected to operation.

The fullest and most elaborate, and perhaps the most accurate report is that given by $\mathrm{Dr}$ George W. Norris in the American Journal of Medical Sciences for October 1849, and an abstract of which was published* in the seventy-third volume of this Journal. Dr Norris remarks at the commencement of this tabular view that the numbers presented, place in a striking light, the dangers attendant upon the Hunterian method of operation for what ap-

* Edinburgh Med. and Surg. Journal, vol, seventy-third. A pril 1850, p. 492. 
pears to the most common form of external aneurism ; and, inasmuch as it is the form of aneurism to which the treatment by compression is more particularly adapted, he subjoins a tabular statement of the cases treated by the latter method, taken from the essay of Dr Bellingham, and such others as had come to his knowledge since the appearance of his performance.

The table of Dr Norris contains two hundred and four cases, in which the femoral artery was enclosed in a ligature according to the Hunterian method. Of these there recovered one hundred and fifty-four; and fifty died. Ten of the patients who recovered had to undergo amputation in consequence of the approach of gangrene of the limbs. Of these 204 cases, one hundred and seventy-seven were instances of aneurism, 155 of Popliteal Aneurism, and 22 of femoral aneurism, in which the operation was performed. The general distribution of results is the following:-

Number of cases,

Died from the effects of the operation, $\quad 38$

Recovered after subsequent amputation, $\quad 6$

Recovered after suppuration of the sac, $\quad$ 10

Recovered after gangrene of the foot, $\quad 2$

177

56

One out of every three cases, therefore, operated upon, either terminated fatally, or were to a certain extent deprived of the use of the limb for the remainder of their lives.

These instances took place in the practice of the most eminent and experienced surgeons; Hunter, Cline, Deschamps, Pelletan, Home, Cuoper, Travers, Lawrence, Brodie, and others too numerous to recount.

The inference from all these facts Dr Norris thinks is, that the method of curing aneurism of the lower extremities by enclosing in a ligature the sound part of the artery above the tumour is not attended with the advantages which it was expected to afford. Sound surgery, according to Dr Norris, condemns this method of treatment.

To demonstrate the superior advantages which this method of compression presents, Dr Norris gives a table of all the recorded cases, in which this method has been adopted since the year $\mathbf{1 8 4 2}$, partly derived from the tract on aneurism by Dr Bellingham, partly from the report on surgery contained in the first volume of the Transactions of the American Medical Association. From this table it results that compression has been tried in thirty cases of popliteal aneurism, and seven cases of femoral aneurism, in all thirty-seven cases; that of these thirty-seven cases only one person died suddenly from disease of the heart forty-eight hours after pressure had been withdrawn; and in one case the compression failed to accomplish the purpose, and the operation was performed. There are therefore thirty-five recoveries in thirty- 
seven cases, or only one death in thirty-seven cases, and one instance in which the compressive method was found inadequate. In one case, the 24th, by Dr Bellingham, pulsation continued in the sac for some time after compression had been applied. A galvanic current was transmitted through it. Seven days after this, the patient was attacked by rose and died after the lapse of six days.

For this method of operation, accordingly, Dr Norris is a strenuous advocate, next after Dr Bellingham; and Mr Tufnell follows both in recommending this method of treating aneurismal tumours as infinitely superior to that by ligature. As the evils for which Mr Tutnell proposes a remedy are of a serious description, and as the remedy has been shown to be at once practicable and adequate, it is right that we should allow the advocates of the method to have a fair hearing with the profession.

From what has been already stated of the history of the operation for the treatment of aneurism, it is clear that the method by compression is not new. It was at one time indeed practised probably much more frequently than the method by ligature. At all events, both from what is taught by Heister and Plattner, two considerable surgical authorities in the first half of the eighteenth century, it is clear that it was very frequently attempted. It appears to have fallen so completely into desuetude as to be much overlooked, only after the introduction of the Hunterian method, and the great names by which that operation was sanctioned and supported. It naturally comes to be an object of inquiry, how, if it possess any merits whatever, it should have been so long in oblivion and abeyance ; and by what means, and under what conditions, it is now revived and placed before the profession, with claims of no moderate urgency to be re-instated in surgical favour.

It was neither in London nor Edinburgh, Manchester nor Liverpool, Paris, nor Vienna, nor Berlin, nor at Rome, nor Florence, nor Bologni,-Madrid need not be mentioned,-that the compressive treatment of aneurismal arteries was raised from the tomb to which, in all appearance, it had been consigned. It was in the eity of Dublin.

It had been long observed by surgeons that in certain cases of aneurisn otberwise favourable for operation, when the operation was performed, gangrene came on, the sac inflamed, suppurated, and sloughed, or some other great catastrophe disappointed the expectations of the operator. It was inferred that this and similar results were occasioned by arresting more rapidly than the collateral circulation could admit, the current of blood through the main channel of the limb. The inference was not unnatural, though we do not think that it was founded on all the circumstances of the case. Right or wrong, however, well founded or not, such was the inference; and, acting upon it, it was believed that if compression by means of a tourniquet or any other manage- 
able instrument, were gradually and gently carried on, the force and amount of the blood sent through the main artery would be so much diminished, while the quantity sent through the small collateral and anastomosing branches would be so gradually yet considerably increased, that for the nutrition of the limb ample provision would be made, and the deligation of the artery, when it came to be performed, would be effected without disturbing materially the circulation of the limb.

Upon this principle chiefly Mr Todd, of the Richmond Hospital attempted compression in a case of aneurism, in the year 1820 . The idea of $\mathrm{Mr}$ Todd is represented to have been that "by compressing the principal artery for a few hours every day, mortification of the limb might be prevented by allowing some progress to be made in establisbing the collateral circulation," - "at the same time that he was not altogether without hope, that, by diminishing the current of blood in the trunk of the artery, so as to favour the coagulation of the contents of the sac, a cure without operation might be effected." In this expectation, huwever, Mr Todd was disappointed. The operation was required and performed.

The next trial of this method was in 1824 , and it was more successful.

A tidewaiter of intemperate habits, who was in 1818 labouring under popliteal aneurism, made a violent exertion, during which the aneurism burst and became diffused. More than one week afterwards, this person applied for relief to Dr Duggan, surgeon to the revenue police of Dublin, who at once informed the patient that amputation was indispensable; and he accordingly removed the limb above the knee.

Six years after this event, namely, in May 1824, the tidewaiter observed in the course of the femoral artery of the stump, one inch and a-half below the ligament of Poupart, a pulsating tumour, which gradually increased in size, and in the following August had attained the magnitude of a Turkey's egg. Dr Duggan, whom the patient again consulted, deemed the operation not advisable, and determined to try the instrument of Mr Todd for compressing the artery in the groin. T'he instrument was applied on the 27th of August ; and on the 20th of October all pulsation had ceased in the tumour. It is not said whether the tumour was much or in any degree diminished. But it is stated that the patient subsequently survived upwards of twenty years.

The following year, namely, in 1825, Mr loodd treated with success an aneurism of the femoral artery by applying steady and forcible pressure over the artery by means of the same instrument.

The instrument or apparatus resembles a common femoral truss or spring bandage, with a stronger spring than that of the hernia truss, the pad longer, of a more oval shape, and more firmly stuffed. The pad of this instrument is furnished with, or rather supported 
and corroborated by an internal plate of iron, which is connected with the outer iron plate, by means of a hinge close to the junetion of the outer plate with the spring; while, by means of a tourniquet screw passing through the place, the attendant has it in his power to make a greater or less degree of pressure by the pad on the part to which it is applied. This instrument was fitted on the limb like a common rupture truss, with the pad applied over the course of the crural artery immediately below Poupart's ligament; and it was effectually retained in its position by means of a strap of soft chamois leather, which passed from the spring of the truss behind on the inside of the thigh, and was buttoned in front, on the outer plate of the pad. A figure of the instrument is given.

$\mathrm{Mr}$ Tufnell is unwilling to admit, that this case, however successful were its termination, and however close might be the connection between the use of the instrument and the result obtained, laid the foundation for compression as an ordinary and established mode of cure. The reason which he assigns for declining to admit this inference, is curious. Mr Todd, he says, soon afterwards died. The case was not published; and the fact of its occurrence within the Richmond Hospital made no impression on his colleagues. The treatment never was introduced. To speak to the exact fact, the example set was not followed.

Seventeen years elapsed, during which the ligature was invariably employed in the Richmond Hospital until October 1842 ; and on that occasion also it would have been the means adopted; but the patient declined to submit himself to the preliminary incisions. Under these circumstances, Dr Hutton, under whose care this patient was, from reading and knowledge of plans, which, from time to time had been proposed or put in use, resolved to attempt the cure of the aneurism by means of compression. This intention Dr Hutton announced to his colleagues, who approved of the proposal, while Mr Adams mentioned the favourable termination which $M r$ Todd had obtained in 1825 from the method.

It is stated that, up to this time, the result of $\mathrm{Mr}$ Todd's case was unknown to Dr Hutton, and indeed to all but Mr Adams, or it had made upon them no impression; and accordingly, Mr Tufnell represents the proposition as perfectly newly devised by Dr Hutton. It is not easy to conjecture what may have been the reasons for this total forgetfulness of the result of Mr Todd's case. It might be, that one case was not thought sufficient to justify a considerable innovation, in what was at that time regarded as an established practice. It seems, nevertheless, neither reasonable nor just to say, that because Mr Todd's experimental case was neglected and forgotten by his colleagues, he had no merit in proving the practicability of the principle which that case implied. That the facts of the case were unknown was not the fault of MrTodd, who had shown 
these facts in a public hospital. It showed only a large amount of indifference, and perhaps some degree of obstinacy, on the part of those by whom the experiment was witnessed. $W_{e}$ can see no reason, therefore, for admitting the conclusion of $\mathrm{Mr}$ Tufnell, that from the case of Dr Hutton must be dated the origin of the present treatment of aneurism by compression, and that this case must be regarded as the foundation of the method. For the facts given by Mr Tufnell himself, if we are not to go back to the times of Dionis, Heister, Plattner, and Guattani, for the first trials of the practice, certainly $\mathrm{Mr}$ Todd is entitled to what ever merit belongs to the revival of the method, since the commencemeut of the nineteenth century.

It must, nevertheless, be allowed, that a considerable degree of merit belongs to Dr Hutton for the second revival of this method of treatment ; for it appears that in consequence of the successful result of the case in which he tried the compressive method in November 1842, other surgeons were induced to subject the method to trial in similar cases.

The instrument or apparatus employed by Dr Hutton is very similar in principle and operation to all the other means, that have been devised with similar intentions. A circular iron hoop one inch and a quarter broad and seven inches in diameter has fitted to one side a screw terminating in a pad. To this pad, and at right angles to the hoop, is attached a small slip of wood one inch broad and nearly five inches long, on the upper end of which is fixed sufficient chamois leather to make a pad, a deep notch being cut on each side of the wood at the other end. When the iron hoop was placed on the thigh as close to the pubis as was possible, the wooden slip pointing upwards, the pad attached to the iron serew was forced down on the vessel. A cord previously tied round the notch in the lower end of the piece of wood fastened it to the pad; and thus secured, it pressed directly on the vessel, while the padded end of the piece of wood took the artery as it passed over the pubis. This apparatus was applied in a case of popliteal aneurism in a man of thirty years on the $3 \mathrm{~d}$ of October 1842, and was kept applied for twenty-eight days with the effect of curing completely the aneurismal swelling.

In January $1843 \mathrm{Mr}$ Cusack adopted the same method with a patient in Steven's Hospital, employing an instrument which Sir Philip Crampton had contrived and employed successfully for compressing the femoral artery at the os pubis in a case of secondary hemorrhage, where the ligature had been applied in Scarpa's space. The principle of this instrument, though differing a little in details, is quite the same as the others. It is sufficient to say, that after its application for the space of thirty-one days, the tumour ceased to show any pulsation and was cured.

The next case occurred in March 1843 to Dr Bellingham, 
who, by means of a similar apparatus, effected in less than fortyeight hours consolidation of a large aneurism in the pophteal space, measuring three inches in transverse and more in the long diameter. This case is regarded as the great triumph of the compressive method of treating aneurism, and not only induced $\mathrm{Dr}$ Bellingham to espouse very strenuously and perseveringly the cause of compression, but has manifestly led all the Dublin surgeons to speak of the efficiency of the method, in those confident terms, which have contributed to furnish so great a number of illustrative and corroborative cases.

The method of maintaining the compression was nevertheless not free from objection. It appears to have been in all cases irksome, and in some it seems to have caused so much pain that the apparatus required to be removed for a time. This was ascribed to the firm and unyielding sort of pressure effected by the screw. By introducing bands of vulcanized India-rubber and compresses of the same, a method devised by Dr Carte, this objection has been either removed or very much diminished. We are to understand, in short, that the new instrument or apparatus as completed according to the suggestions of Dr Carte, is the standard of perfection for compressing an artery in attempting the obliterating of an aneurismal tumour or the adhesion of the arterial walls for any other purpose. This apparatus is in surgery what the lines of Villars from Bouchain to Arras, or those of Torres Vedras were in the military art.

It is necessary further to mention that, besides the mere artery compressing apparatus, there is another auxiliary or vicarious method strongly recommended, first by $\mathrm{Dr}$ Bellingham and again by $\mathrm{Mr}$ Tufnell. This consists in the use of a weight of a conical shape, averaging from six or seven to ten pounds, with a small oval pad attached to its small end. The weight is to be placed upon the artery in the groin, and there retained by the patient's hand. The use of this, however, is admissible only at one spot of the artery, that, namely, where the vessel crosses the os pubis, and where a bony point for counter-pressure can be rendered a vailable in the same manner. Though the means be simple, $\mathrm{Mr}$ Tuffnell looks upon it as important and efficacious.

Our limits permit us not to enter into the descriptive details of either the improved apparatus of Dr Carte, or the multiplied contrivances by which it is sought to attain the same object. Of all the principle and end are the same; namely, to compress more or less forcibly the femoral artery in the groin, and thereby to diminish and cut off the flow of blood through the femoral artery, to produce obliteration of its channel, adhesion of its walls, and the consequent shrinking and obliteration of the walls of the aneurismal sac. Mr Tufnell speaks in terms of high commenda- 
tion of the apparatus of Dr Carte; and we doubt not that the terms are merited; and he is not less decided in representing all the other apparatus as altogether useless, inefficient, and already obsolete, and antiquated. "The others," he says, "are all obsolete; they have had their day, and gone, giving way to the improvements made in those I name-alterations calculated to obviate deficiencies existing before." -P. 56.

The other contrivances here referred to are partly those already mentioned, partly six other forms of apparatus; namely, Read's Pelvic saddle for compressing the artery at the groin,- the ring Tourniquet, and the Clamp,-L'Estrange's Tourniquet,-Millikin's double-padded Compressor, - and the double-actioned Compressor. Of these the author gives figures; and he allows that the forms of the instruments may prove useful in the adoption of such modifications and alterations, as may be requisite to suit particular cases. But he expresses confidently the opinion that he considers none as calculated for the treatment of aneurism by compression, that are worked solely on the principle of the screw.

Very much inclined would we be to assent to all this exclusive commendation of one apparatus, had we not lived to see the most excellent and suitable apparatus for various purposes in surgery completely thrown aside, after the lapse of some years; and seen preserved in museums and other collections of curiosities, or thrown into the lumber rooms of hospitals, as no longer useful, mechanical contrivances upon which, in their day, the same judicious commendations had been pronounced. Even for the very purpose of compression, there are delineated and described in some of the not very recent systems of surgery - for instance, Dionis, Heister, and Plattner, - pieces of mechanical apparatus which we arc assured by respectable and credible authorities, admirably answered the purposes intended; and which, notwithstanding, are now altogether unknown except to antiquarians in surgery and collectors of surgical curiosities. To the observer of modes in surgical apparatus, it will be a matter of interest to notice and record what has become of all these contrivances, not excluding that of Dr Carte, in the years 1861 and 1871.

Such in plain and unadorned language, - avoiding the magnificent expressions of the eloquent Mr Tuffnell,-is the history of the introduction or rather revival of the method of treating aneurism by means of compression.

On the question as to the effects of compression, the knowledge of which furnishes the only method of determining when it ought to be employed, or whether it ought to be employed at all, $\mathrm{Dr}$ Bellingham has made some instructive observations. This author first establishes from various facts the following aphorisms.

VOL. LXXVI. NO. 188. 
1st, That nature herself sets up a process by which, under favourable circumstances, the cure of aneurism is effected.

$2 \mathrm{~d}$, That the mode in which this is effected always in internal aneurism, and frequently in external aneurism, is by the deposition of the fibrine from the blood in the sac of the aneurism, until it becomes filled.

$3 \mathrm{~d}$, That the fibrin in such cases is deposited in regular concentric laminae, the oldest or first formed next the sac, those most recently formed nearest the centre.

4th, That a current of blood through the aneurismal sac is a necessary agent in bringing about this result.

5 th, That any obstruction to the current by which its velocity and amount are diminished, will accelerate the deposition of fibrin in the aneurismal sac.

6 th, That when once this process has been commenced, if the same agents continue in operation, it will go on until the sac becomes filled, and no longer permits the entrance of blood.

Dr Bellingham is further anxious to correct several erroneous but generally received notions on compression, and its effects. Though surgical writers have generally appeared to think, that in order to effect the cure of an aneurism by compression above the tumour, it was necessary to interrupt completely the current of blood through the vessel, in short, to apply pressure which would act in a manner equivalent to the ligature, induce inflammation in the arterial coats, and obliterate the circula. tion in the vessel at the point to which the compression was applied, Dr Bellingham infers, that it is not at all essential that the circulation through the vessel leading to the aneurism be completely checked, but rather the contrary. He allows that this degree of compression may be advantageous at first for a short period, as by that means the collateral circulation will be more certainly established. The result of one case, which was communicated by Dr Bellingham to the Surgical Society of Ireland, at a meeting held on the 22d April J843, establishes, he infers, the fact, that a partial current through an aneurismal sac will lead to the deposition of fibrine from its interior, and cause it, within a few hours, to be so filled and obstructed as no longer to permit the passage of blood through the sac.

Dr Bellingham, in short, draws two inferences which may be regarded as the foundation of the whole theory. The first is, that it is not by the formation of a coagulum in the aneurismal sac that nature effects the cure of the disease, but by the deposition of fibrine from the blood, which circulates through the sac. The second inference is, that simply diminishing the current will not cause the coagulation of the contents of an aneurism, but it will occasion the depasition of fibrine in the sac. 
sere is a strong temptation in this case to ask what is the serence between causing the coagulation of the contents of an aneurismal sac and the deposition of fibrine within the sac? Is fibrine deposited without coagulation; is not the deposition of fibrine a species of coagulation? If coagulation take place, is flbrine not deposited? We must admit our inability to perceive the distinction here attempted to be made. If the contents of an aneurism are coagulated, we think that the deposition of fibrine has either already taken place, or must speedily follow. All this, nevertheless, we submit in deference to Dr Bellingham, who probably has some mode of showing the difference between the two processes. Let it be remembered, that we have read what he states at p. 137.

It is right nevertheless to allow Dr Bellingham to state his facts in his own way:-

"When the sac of an external aneurism has become filled by fibrine, the disease is evidently also cured, and the circulation through the artery may continue for a time; but the anastomising branches here being numerous and free, the blood passes down the main artery of the limb in a dimished stream, the deposition of fibrine therefore continues until the artery at the seat of the aneurism is encroached upon and gradually filled by fibrine, when its pulsation stops, and the deposition of fibrine ceases.

"Once a certain amount of fibrine has been deposited in an aneurismal sac, as the result of pressure upon the artery above, there would appear to be an irresistable tendency to its continuing to be deposited until the artery at the part is likewise closed up; this fact has been proved by the two cases to which I have recently referred, where the patients refused to continue the pressure, and commenced taking exercise; yet under these apparently unfavourable circumstances, the pulsation ceased after a short interval, and the cure was effected. It is therefore not unlikely that the artery has many times been tied in aneurism, where the process which nature sets up for its cure had made considerable progress, and where a little further delay would probably have done away with the necessity for its performance.

"The ultimate changes which takes place when the pulsation of an external aneurism ceases, are the same, whether this result has occurred spontaneously or been brought about by compression. The circulation is carried on by the enlarged collateral vessels, the contents of the sac and of the artery at the part from which it springs, are gradually removed by the absorbents, the sac disappears in a great measure, and the vessel is eventually converted into an impervious ligamentous band. Guattani, Petit, besides many other writers, have reported cases of aneurism spontaneously cured, where this condition of the parts was found; and I have already described the appearances which $I$ found in a case where the patient died two years and a-half after the cure of a popliteal aneurism by compression, and sixteen months after the cure of a femoral aneurism in the oppcsite limb; 'the artery at the site of each aneurismal sack was con- 
verted into solid, thick, ligamentous band, its channel was ob. rated, and the contents of the sacs had been removed by the absor. ents.

"The foregoing facts all go to prove, that when a cure of external aneurism takes place spontaneously, or when it is brought about by compression, it cannot fail of being permanent; the sac is filled up, and the artery from which it springs is obliterated; consequently, pulsation cannot return, nor can a secondary aneurism form at the part, both of which have occasionally occurred after an apparent cure by the ligature."-Pp. 141, 142.

Dr Bellingham subsequently enters into a lengthened disquisition to demonstrate the advantages of the trcatment by compres. sion over that by ligature, and to answer all reasonable objections. As our space will not permit us to follow him over this lengthened course, it may be well to subjoin the following aphorisms as containing a summary of the doctrine which he advocates.

61. The arteries to which compression is applicable being far more frequently the subject of aneurism than those to which it is inapplicable, compression is calculated to supersede the ligature in the great majority of cases.

" 2 . The cure of aneurism by compression upon the artery between the aneurismal sac and the heart, according to the rules laid down here, is accomplished by the gradual deposition of the fibrine of the blood in the sac, until both the latter and the artery at the part are completely filled. The process is in fact exactly similar to that by which nature effects a spontaneous cure of aneurism.

" 3 . Such an amount of pressure as would cause inflammation and adhesion between the opposite sides of the artery at the point compressed is never required.

"4. The pressure should not be so great as to interrupt the circulation in the artery at the point compressed; an essential agent in the cure being that a current of blood should pass through the sac.

“ 5 . Compression by means of two or more instruments, one of which is alternately relaxed, is much more effectual than by any single instrument, and in many intances the pressure can be inaintained by the patient himself.

“ 6 . The treatment of aneurism by compression does not involve the slightest risk to the patient, and if persevered in cannot fail of effecting a cure.

"7. A cure of aneurism effected by compression, according to the laws laid down here, must necessarily be permanent; and in every case in which a cure has been accomplished, the patients have remained well subsequently.

"8. The femoral artery remains pervious after the cure at the point at which the pressure had been applied, and no morbid change of any kind is to be detected in either the artery or vein at the site of the compression.

" 9 . When a cure is effected by compression, the vessel is obli- 
terated only at the seat of the aneurism, and the artery at this-part is eventually converted into an impervious ligamentous band.

"10. Compression effects the cure of aneurism by more simple and safer means than the ligature, while it is applicable to a number of cases in which the operation is contra-indicated or inadmissible.

"11. Compression is not necessarily a more tedious or more painful method of treating aneurism than the ligature, while it is much more certain, more likely to be permanent, and is free from all danger.

" 12. Compression, according to the rules laid down here, has little analogy with the old method which went by this name; and in fact has no greater resemblance to it than the Hunterian operation had to the operation for aneurism which it superseded." - Pp. $180,181$.

Mr Tuffnell is at some pains to impress the necessity of accurate diagnosis of the exact nature of the case before measures for compression be adopted; he mentions the possibility of mistaking rheumatic stiffness and pain for that of aneurism, and the converse; and he quotes one instance in which an enlarged bursa assumed the appearance of popliteal aneurism. He gives the usually received diagnostic marks; none of which can be regarded as characteristic except the stethoscopic murmur; but his exhortations are certainly by no means useless or unnecessary. The following observations are entitled to much attention.

"They are, however, not the only kinds of pain that accompany aneurism in the popliteal space. There is another of a more lasting and boring description. Like the former, (sharp flying pains seening to encircle the patella,) it occurs mostly at night ; but instead of being sharp and flying, it is persistent to a degree often inducing the patient to keep the limb considerably bent. This is an ill omen. It betokens a condition, which, unchecked, leads on to softening and erosion of the posterior facets of the condyles of the femur and cartilage. On the corresponding portion of the tibia, with irritation of the synovial membrane from pressure against the posterior aspect of the joint. It tells us that the aneurism springs from the arterior aspect of the artery, or that nearest to the bone,-a position which, in its subsequent enlargement materially effects the progress of the disease, by inducing it to press against and open into the joint. Such has been the state of things found to exist in cases where the amount of constitutional disturbance rendered either ligature or compression undesirable, and pointed to the necessity for the removal of the limb."-P. 39-4.0.

To this persistent pain, then, Mr Tuffnell directs particular attention, because he is of opinion that it may tend materially to assist in forming a diagnosis, and prevent the surgeon from offering too favourable an opinion as to the result of the case. Aneurism, in sliort, when arising from the articular or anterior 
aspect of the popliteal artery is a more formidable malady, than when it springs from the posterior or lateral portion of the vessel, and requires to be checked in its progress with the least possible delay.

The cure, he also thinks, is less speedy in the case of fusifurm than in that of the globular-shaped sac.

Aneurisin of one of the external arteries, or those of the extremities, is occasionally associated with aneurism of some internal artery; the arch of the aorta, the innominata, the descending aorta. In cases in which there is any reason to believe in the existence of this complication, Mr Tuffnell pronounces the treatment by ligature altogether inadmissible, as likely to be most hurtful, and the method by compression as alone calculated to effect a cure, ( $p .44$.

Compression he further considers to be applicable in every case of ordinary circumscribed aneurism, in an extremity where there is sufficient room for the application of the compressing agent at two different points above the tumour, providing the pressure on the trunk of the vessel completely controls pulsation in the sac, thus proving that no high bifurcation exists.

Conversely, Mr Tuffnell does not advise it in cases, in which the aneurismal tumour is rapidly enlarging in size, or where it continues to do so after compression has been attempted. Aneurisms of this kind have no distinct sac; and to afford any chance of saving the limb, the blood, through the main channel, must at once be cut off by enclosing the ressel in a ligature. Neither does he think it advisable in cases, in which the disease has been allowed to advance unchecked, where the limb has become odematous and swelled, and the surface of the tumour has assumed a dusky yellowish red colour. In such circumstances he thinks that most probably the vein is involved in the disease; and if it be popliteal aneurism, the knee-joint is inflamed. Under such circumstances, amputation he regards as the only resource.

It is further necessary, Mr Tuffnell observes, to consider the state of the system in which aneurism may occur. Though, generally speaking, it is a malady taking place in persons of muscular development, and apparently healthy frames, yet occasionally it manifests its presence in persons of nervous habit and anaemic disposition. In cases of the former order, or the hyperemic, it is important to quiet the circulation by rest, restricted diet, the occasional use of saline purgatives, and compound jalap powder, with cream of tartar solution for drink, and, if need be, bloodletting, and the use of foxglove. For the two latter remedies, however, he thinks that occasion is seldom requisite. In the nervous and anaemic state, on the other hand, chalybeates must be administered, and the strength cautiously supported.

When the instrument or apparatus has been applied and kept 
retained over the trunk of the affected vessel for some time, several days, twenty days, twenty-five days, or even longer, the first important change that takes place is, that the outline of the sac beconies more distinctly defined and circumscribed. When pressure is relaxed, the tumour pulsates less strongly than formerly, and the patient may be sensible that he cannot feel the sac distended by the influx of blood as plainly and as painfully as it was at first. The whizzing murmur becomes much softer and emits a less roughened sound.

When these changes are taking place, great care is necessary, as a few hours are often sufficient to produce consolidation of the whole contents of the sac. The case should therefore be watched; and if the circulation through the aneurism have not been previously completely controlled, it should now be stopped altogether. This is soon succeeded by flying pains in the tumour, which shoot from it down the outside of the leg, and run over the toes and foot. These are represented to be often preceded by nausea and a serisation of debility throughout the whole frame, in short, a certain amount of symptoniatic fever. After this, in a certain number of cases, follows a burning pain within the tumour and throughout the limb, often waking the patient if he happen to be asleep. Occasionally, on the other hand, in rapid cases, as well as those of more gradual course, the pulsation finally ceases, and no such occurrence takes place.

Mr Tufnell enters into some details regarding certain peculiarities taking place in particular cases. For these, however, the reader will do well to consult the work itself.

According to the method of treatment thus strenuously recommended by Dr Bellingham and Mr Tufnell, thirty-nine instances of popliteal, femoral, brachial, and radial aneurism have been treated, all in Dublin, either in public institutions or private houses, during the space of eight years, extending from October 3d 1842 to February 1851 . The following are the results:-

In thirty of these cases the cure has been perfect and complete.

In one case (20) compression was discontinued, the aneurism not subsequently increasing in size.

In two cases (10 and 25) the ligature was resorted to, and the artery was tied with success.

In three cases $(26,38)$ amputation became necessary, each instance being followed by recovery.

In one case, death ensued from erysipelas.

In two instances death took place in consequence of co-existing disease of the heart.

The forms of aneurism, in reference to situation, were twentyeight instances of the popliteal artery, seven of the femoral, three of the brachial, and one of the radial artery. 
Of the whole thirty patients twenty-eight were males, only two were females. In one of the females the disease was traumatic aneurism of the brachial artery caused by gunshot.

Of the male patients, one was eleven years, another fifty-five, another forty-eight. All the others, in number thirty-four, were from twenty-five years of age to forty-four inclusive; but at least twenty-eight were below thirty-five years.

Regarding the time during which pressure was applied, the shortest case of all is one by $\mathrm{Dr}$ Hutton, in which the cure was effected by compression for seven hours ; and one by Dr Humfrey, in which it was effected after ten hours. Next comes one by Sir Philip Crampton, sixteen hours; one by Dr Bellingham, twenty-three hours; one by Dr Humfrey, thirty-three hours; and one by Dr Clayton, thirty-nine hours.

Next come the cases in which the time is computed by days. First comes that by Dr Bellingham, already mentioned as only two days, and a case by Dr Macdonnel, in the Richmond Hospital, also two days. One case by Mr Cusack, is four days; one by Dr Bannerman, five days; one by Mr Tufnell, six days; one by Mr Cusack, seven days ; one by Mr Cusack, nine days; one by Dr O'Ferrull, eleven days; one by Dr Hutton, twelve days; and one by Mr Tuffrell, thirteen days.

After this the cases rise to twenty days, of which there are three examples ; twenty-one days, two instances; twenty four days; twenty-eight days; thirty-one days; thirty-three days; thirty-seven days, forty-two, and forty three days, fifty-three days, seventy days, seventy-two days, and ninety-three days.

From these numbers not much serviceable information can be elicited. All that they show is, that, though in certain favourable cases pressure over an aneurismal artery for seven, ten, or sixteen hours may be followed by obliteration of the channel of the artery and contraction of the aneurismal sac, yet this can be rarely expected in a shorter space of time than twenty days, and in certain cases it will occupy twice or three times that period. The average of the whole is in round numbers twenty-five days.

We do not think, nevertheless, that with all these allowances and deviations the treatment can be called wearisome and prolonged. The average time for the treatment of the cases, $\mathbf{M r}$ Tuffinell calculates at twenty-four days and twenty-one hours, or in round numbers twenty-five days; and assuredly for getting cured of a disease so certainly fatal, without surgical interference so often fatal even with that, it is worth while for the patient to endure pressure upon the femoral artery for this length of time.

It is justly remarked by $\mathrm{Mr}$ Tuffnell, that if this length of time be contrasted with that required for the cure of deligation, the method by compression possesses many advantages over the latter. 
From the hour or day that the patient with aneurism is admitted into hospital, till that in which the operation can be performed, in general one week must elapse. From the date of the application of the ligature to that of its detachment sixteen days longer must be given; and for closure of the wound and cicatrization, five days more may be allowed, making altogether twenty-eight days, under the most favourable circumstances, if there being no hemorrhage, no inflammation or sloughing or laceration of the sac, in short, no unfavourable accident. If it be remembered in how many instances of deligation, some of these catastrophes take place, or amputation requires to be performed, to give the patient* a slight chance for life, few will hesitate to assign the preference to the method of treatment by compression.

That surgeons will ultimately adopt the method by compression for treating femoral and popliteal aneurism, Mr Tufnell speaks in the most confident terms. He entertains no doubt that it will become the general method of treatment. Truth, he says, ever has prevailed and ever will prevail. This is all very well, if in this obscure, confused, and uncertain state of existence, we knew and could tell what is truth. But as this is not likely to be the case in the course of the present age, we must leave the matter to be determined in the usual way. None can doubt, that, as the matter at present stands, it must be a great improvement to have a systematic and safe method of applying pressure in the case of arteries going to aneurismal tumours; and Dr Bellingham and Mr Tuffnell have certainly made out a strong case for the adoption of this method in the treatment of aneurisms affecting the lower extremities.

Dr Bellingham has examined with great learning and knowledge the question of the treatment of aneurism, and has adduced strong facts and arguments against the use of the ligature, and in favour of the method of compression. Mr Tuffnell has also collected a large body of evidence against the ligature and in favour of compression. Both authors, however, have overlooked in their investigation, certain circumstances, which, in our opinion, are so important, that no investigation can be complete without them. Both authors have shown by a plentiful induction from numerous facts that the application of a ligature to an artery, which has in its course an aneurismal tumour, is an operation which is in a large proportion of cases pregnant with pernicious consequences. They do not, however, inform the reader of the reasons, why it is so. Now this we conceive to be the great point for the surgeon to understand. An artery affected with aneurism is always an artery in a state of considerable disease. Its coats both above and below the aneurismal tumour, are either in a state of chronic inflammation, or present the effects of chronic inflamVoL. LXXVI. No. 188 . 
mation, or they are in a state of sub-acute inflammation; the entire circulation and nutrition of the arterial tissues is completely deranged. No artery becomes aneurismal until it has been long previously in a state of ehronic disease; steatomatous deposition, or what Dr Gulliver has shown to be fatty degeneration, osseous degeneration, and atheromatous disintegration. Then the aneurismal sac and the arterial coats above and below it are invariably reddened, softened, always brittle, sometimes lined with false membrane. We never yet dissected a case of aneurism either of the innominata or the arch of the aorta, without seeing the whole of the branches arising from them, all reddened, softened, and covered by false membrane; and invariably during life there is much pain along the course of these vessels. It is the same with aneurisms in the arteries of the extremities, excepting the single case where the aneurismal tumour is caused by a punctured wound. Examine also the course of these arteries, and invariably they are the seat of pain and uneasiness; they, in short, are the seat of a process of inflammation. In those instances, not very frequent we allow, in which death has taken place, either in consequence of aneurism of the extremities, or after the operation, the artery has been invariably found in a most unhealthy state. Rigid, thickened in the coats, with patches and rings of bone, with steatomatous and atheromatous deposit, the inner coat reddened, thickened, and softened, and peeling off from the middle coat, abrasions in various spots, the middle coat itself presenting steatomatous or waxy-like deposits, the bifurcations bulging out, while the channel and calibre of the artery is contracted and narrowed;-such are the common changes in arteries in the course of which is situate an aneurismal sac. Then what is the aneurismal sac itself? Is it not a mass of arterial tissues, brittle, torn, and utterly void of their natural qualities of elasticity, tension, and resistance. They no longer yield to contract again, but they give way by laceration. They no longer contract and expand according to the quantity of blood sent through them.

Besides this condition of the arterial tubes and their component tissues there is an unsound state of the blood. In this fluid the alkalescent predominance is subverted; it contains acids, sometimes one, sometimes another, in excess; and the effect of the presence of these acids, the resuit of irregularities in diet, excess and indulgence, and sometimes of mercurial courses, is not only to render the blood more prone than natural, to form ceposits albuminous, fatty, and saline, but to irritate to a great degree the internal tunic of the whole arterial system. Conceive blood charged with much more carbonaceous matter than it ought to have, with uric acid, lactic acid, phosphoric acid, besides lime and other articles beyond the normal amount; conceive alkohol and various other articles absorbed and circulating, or, at all events, irritating 
and poisoning this blood; and then some idea may be formed of the original causes of aneurismal arteries.

$\mathrm{Mr}$ Tuffnell speaks, at page 32, in a strange manner of aneurism being generally supposed to be a disease slow in formation, slower at least, he says, than his own experience would lead him to believe. The inference from this is, that aneurism is a disease rapid in formation; and in proof of this he refers to an instance in which the artery in the form gave way, and thirteen days afterwards, the tumour, he adds, was the size of an egg ( $p 33$ ). He afterwards notices the occurrence of a case which took place in the course of eight days, and in which the tumour increased in the course of forty hours to the magnitude of Florence flask ( $p 34$ ).

It is not easy to answer for all the irregularities of surgical doctrine, and all the fancies in surgical pathology. But we cannot imagine any sensible surgeon of the present day believing, that the pathological circumstances which determine the production of aneurism, are rapid in course and recent in date. The arterial coats must have been long previously in a state of chronic disease, with occasional inter-cufrent attacks of acute or subacute character. It is under these conditions that the tumour, which is a mere effect of the disease, is formed, and increases according to circumstances, rapidly or slowly. A sudden jerk or wrench, incautiously putting the foot down, a leap, unguardedly and suddenly extending the leg upon the thigh, may produce in one instant in such an artery an amount of laceration which will most speedily attain a great magnitude.

The practical deductions from all these facts are, that not only is the ligature not by any means so rational or safe a measure as was at one time thought, but even compression is itself and alone calculated to do mischief. Conpression, nevertheless, must be allowed to a certain extent, to be a move in the right direction. But it proceeds not sufficiently far. No method can be expected to be serviceable in the treatment of aneurism, which does not combine the use of those measures which are calculated to remove, so far as they are removable, the morbid conditions of the arterial coats. The surgeon will require to adopt practice much more decidedly medical than he has hitherto done, if he wish to obtain favourable results even from tho use of compression. The great object which he ought to keep in view is to abate and diminish inflammation along the course of the affected artery, to put a stop to all sources of irritation, and to place the circulation, general and local, in a state as tranquil as possible. It is assuredly a great difficulty in the way of rational treatment, that patients with aneurism have generally been persons intemperate in labits, sensual, incapable of self-denial, or practising any of those restraints, which are not less useful to the health of the body than the er ergy of the mind. Here, therefore, the surgeon must meet 
with difficulties and iupediments. At the same time, if he keeps in view the fact, that there is in the frames of these persons a general inflammatory state of the whole arterial system, probably a morbid condition of the blood, and a local inflammatory condition of one artery and set of vessels, he will see that, unless these conditions be removed or mitigated, he can expect beneficial effects neither from compression nor from any other mechanical contrivance. The medical treatment of aneurism, we must repeat, is by far the most important part of the duty of the surgeon; and before ten years more have elapsed, the truth of this principle will be recognized and avowed.

\section{ArT. III.-Elements of Chemistry, Theoretical and Practical,} including the most recent Discoveries and Applications of the Science to Medicine and Pharmacy, to Agriculture, and to Manufactures. Illustrated by Wood-cuts. Second Edition. By Sir Robert Kane, M.D., M.R.I.A., President of the Queen's College, Cork, Director of the Museum of National Industry, Member of the Society of Pharmacy of Paris, \&c. \&c. \&c. Dublin, 1819, 8vo, pp. 1069.

THis work made its first appearance at Dublin in 1841 ; and it was in no long time recognised to be an excellent and serviceable treatise on the elements of Chemistry. It was indeed well received, not only in Ireland, but also in the United States.

The present is the second edition, and sustains with ability the character established by the first. It may be described as a judicious and faithful compendium of the doctrines, principles, and facts, of which chemical science at the present time consists. The author gives just views on the great leading doctrines; he gives a correct history of the different chemical elements and compounds, and their reactions; he takes care to show the application of chemical principles and chemical and physical facts in explaining the phenomena of the material world; and the phenomena and actions of living beings, vegetable and animal; and he has given full and correct accounts, so far as the limits of this work allow, of the chemical history of organic bodies generally. He shows also the applications of chemistry to various arts ; glass-making, metallurgy, and agriculture.

We have perused the work with much pleasure,-many parts of it more than once; and upon the weright of this experience, we recommend it, not only to the student of chemical science, but to all those, whether professional or general, who wish to form just and enlarged ideas on the nature, purposes, and serviceable applications of chemistry and the chemical arts. 